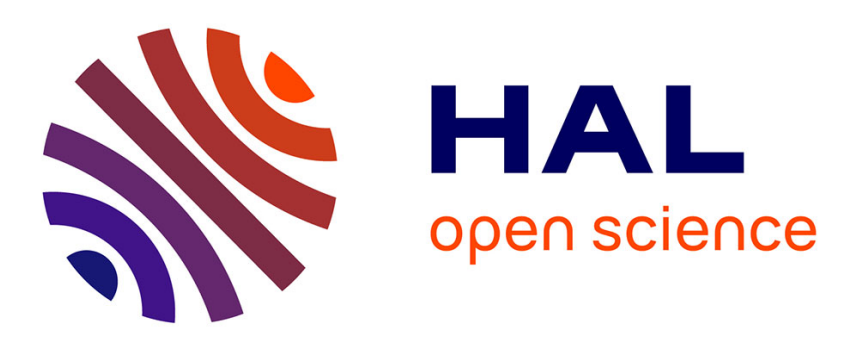

\title{
Real-Time Nonlinear Model Predictive Control of a Glass Forming Process Using a Finite Element Model
}

\author{
Janko Petereit, Thomas Bernard
}

\section{To cite this version:}

Janko Petereit, Thomas Bernard. Real-Time Nonlinear Model Predictive Control of a Glass Forming Process Using a Finite Element Model. 25th System Modeling and Optimization (CSMO), Sep 2011, Berlin, Germany. pp.266-275, 10.1007/978-3-642-36062-6_27 . hal-01347546

\section{HAL Id: hal-01347546 \\ https://inria.hal.science/hal-01347546}

Submitted on 21 Jul 2016

HAL is a multi-disciplinary open access archive for the deposit and dissemination of scientific research documents, whether they are published or not. The documents may come from teaching and research institutions in France or abroad, or from public or private research centers.
L'archive ouverte pluridisciplinaire HAL, est destinée au dépôt et à la diffusion de documents scientifiques de niveau recherche, publiés ou non, émanant des établissements d'enseignement et de recherche français ou étrangers, des laboratoires publics ou privés.

\section{(c)(1)}

Distributed under a Creative Commons Attribution| 4.0 International License 


\title{
Real-Time Nonlinear Model Predictive Control of a Glass Forming Process Using a Finite Element Model
}

\author{
Janko Petereit and Thomas Bernard \\ Fraunhofer Institute of Optronics, System Technologies and Image Exploitation \\ IOSB, Fraunhoferstr. 1, 76131 Karlsruhe, Germany, e-mail: \{Janko.Petereit, \\ Thomas.Bernard\}@iosb.fraunhofer.de
}

\begin{abstract}
The control of complex forming processes (e.g., glass forming processes) is a challenging topic due to the mostly strongly nonlinear behavior and the spatially distributed nature of the process. In this paper a new approach for the real-time control of a spatially distributed temperature profile of an industrial glass forming process is presented. As the temperature in the forming zone cannot be measured directly, it is estimated by the numerical solution of the partial differential equation for heat transfer by a finite element scheme. The numerical solution of the optimization problem is performed by the solver HQP (Huge Quadratic Programming). In order to meet real-time requirements, in each sampling interval the full finite element discretization of the temperature profile is reduced considerably by a spline approximation. Results of the NMPC concept are compared with conventional PI control results. It is shown that NMPC stabilizes the temperature of the forming zone much better than PI control. The proposed NMPC scheme is robust against model mismatch of the disturbance model. Furthermore, the allowed parameter settings for a real-time application (i.e., control horizon, sampling period) have been determined. The approach can easily be adapted to other forming processes where the temperature profile shall be controlled.
\end{abstract}

\section{Introduction}

The control of complex forming processes (e.g., glass forming processes) is a challenging topic due to the mostly strongly nonlinear behavior and the spatially distributed nature of the process. The nonlinearity is caused on the one hand by the physics of the process (e.g., radiation) and on the other hand by nonlinear material properties (e.g., memory effect). In recent years considerable progress has been made in the field of simulation tools (e.g., finite element based simulation of complex rheological processes [11], [1]) and model order reduction (MOR) methods [4], [3]. Consequently, the use of these (reduced) models for process control and optimization is in the focus of actual research activities [2]. Nonlinear Model Predictive Control (NMPC) concepts play an important role in this context, as NMPC can be applied for nonlinear and spatially distributed dynamic systems. Main advantages of NMPC approaches are that the 


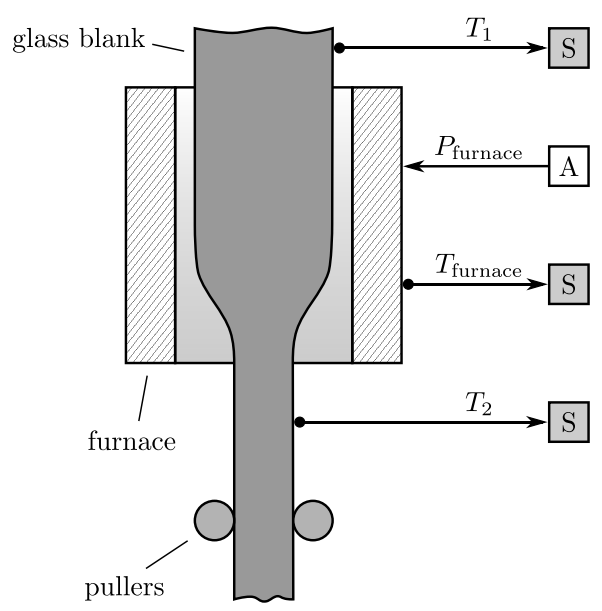

Fig. 1. Glass forming process overview

performance criteria can be designed in a transparent way and that even time dependent constraints can be applied to manipulated, output and state variables of the controlled system. Drawbacks of NMPC are that computational costs for the solution of the nonlinear programming problems often are high, hence powerful optimization solvers are needed [8], [9]. A suited solver for large scale dynamic optimization problems is HQP (Huge Quadratic Programming, [10]). HQP has been used e.g., for NMPC appplications in process industry [12], power plants [7] as well as for optimal management of water resources [5].

For complex forming processes in many cases an optimal spatially distributed (and sometimes time-dependent) viscosity profile of the material has to be assured. As viscosity is difficult to measure on-line, temperature often is used as an auxiliary controlled variable. Hence the problem consists in controlling a spatially distributed temperature profile. In [6] a first NMPC concept has been investigated. In this work a predictive functional control scheme (PFC) has been applied in order to optimize the forming control loops, but not the temperature control. Only a very short prediction horizon is assumed in the mentioned simulation studyy. In the present paper a new approach for the real-time control of a spatially distributed temperature profile is presented. As the temperature in the forming zone cannot be measured directly, it is estimated by the numerical solution of the partial differential equation (PDE) for heat transfer by a finite element (FE) scheme. As the dimension of the state space model, which is result of the FE algorithm, is too large for real-time optimization, in each sampling interval the full finite element discretization of the temperature profile is reduced considerably by a spline approximation. The numerical solution of the optimization problem is performed by HQP. The approach can easily be adapted to other forming processes where the temperature profile shall be controlled.

The paper is organized as follows. Section 2 describes an industrial glass forming process to which the NMPC approach is applied. The details of the 
proposed NMPC concept are presented in section 3. In section 4 the results of the NMPC concept are discussed. Section 5 gives some conclusions.

\section{Optimal temperature profile of an industrial glass forming process}

The NMPC concept which will be introduced in the next section has been applied to an industrial glass forming process (Fig. 1). A vertically hung glass cylinder is fed into a ring-shaped furnace where it begins to liquefy. It then starts flowing downwards viscously and is taken up by pullers which pull the resulting glass tube with an appropriate speed. It is essential for the process to stabilize the viscosity profile along the cylinder. The viscosity can be controlled by the furnace temperature.

Figure 1 depicts the relevant sensors and actuators regarding the temperature control: As the furnace tightly encloses the glass cylinder, there are only three temperature sensors available - one each right above $\left(T_{\text {cyl }}\right)$ and below $\left(T_{\text {tube }}\right)$ the furnace, and one which measures the furnace temperature $\left(T_{\text {furnace }}\right)$ itself. There is no possibility to measure the temperature in the forming zone $\left(T_{\text {form }}\right)$ directly. Thus, a strategy is needed to reconstruct this control variable using the measurements of the only three available sensors. This is achieved by a finite element model. The forming temperature is controlled by increasing or decreasing the heating power and hence furnace temperature.

In order to ensure optimal product quality there are three major variables which must be controlled in an optimal way. The first two are the feeding and pulling speed (respectively force) which are accounted for by a common control loop. We call this one the geometry control. The third variable is the (spatially distributed) temperature of the glass in the forming zone, whose control is done by a seperate loop, which we call the temperature control. In the scope of this paper we will concentrate on the latter, i.e., the temperature control.

For glass the system's behavior is in general strongly nonlinear due to the impact of radiation. The spatio-temporal temperature distribiution can be calculated solving the heat transfer PDE. If a symmetric cylinder is assumed and radial temperature distribution the following one dimensional PDE describes the temperature distribution $T(z, t)$ along the vertical z-axis:

$$
\rho c_{p}(T) \frac{\partial T}{\partial t}=\lambda(T) \frac{\partial^{2} T}{\partial z^{2}}+\dot{q}_{\mathrm{conv}}(T, z)+\dot{q}_{\mathrm{rad}, \mathrm{oven}}(T, z)+\dot{q}_{\mathrm{rad}, \mathrm{dist}}(z, t)
$$

Radiative heat transfer exchange between the oven and the glass is described by the nonlinear term $\dot{q}_{\text {rad,oven }}$ (Stefan-Boltzmann law). Radiation inside the glass is considered by means of an effective heat conduction coefficient $\lambda(T)$ which is nearly exponential increasing with temperature and hence introduces a second nonlinearity in the model. The term $\dot{q}_{\text {conv }}$ describes the convective heat transfer due to the movement of the glass cylinder. A further nonlinearity is introduced by temperature dependence of the specific heat capacity $c_{p}(T)$. 
At the end of a batch production (i.e., the when the length of the cylinder which is fed on the oven tends to zero), disturbances arise due to radiation effects which are caused by the end of the cylinder. This radiative disturbance are described by the space and time dependent term $\dot{q}_{\text {rad,dist }}(z, t)$. As the disturbance cannot be measured directly, it is estimated online by a special parameter estimation approach.

Due to the transport length and the corresponding dead time it is important that the disturbances are suppressed as early as possible by an optimal strategy of furnace temperature during process end phase. As the steadiness of the viscosity in the forming zone exerts the main impact upon the quality of the final product, an optimal control must stabilize the spatially distributed temperature (and hence viscosity) profile of the forming zone as good as possible.

To accommodate for the deficiencies of the PI controller we developed a control concept which explicitly takes into account the large dead time of the process and the disturbances arising during the end phase. As a PI controller can only react to changes in the control variable which are already in effect, we switched over to a predictive control strategy, which is able to take corrective action before any impact of the disturbances is visible to the temperature sensors. This behavior is necessary since the delay time of the furnace is too large to lower the temperature in sufficient time. Otherwise, overshooting of the forming temperature would be unavoidable.

As the prediction model for the NMPC scheme we exploit the finite element model of the $1 \mathrm{D}$ temperature distribution $T(z, t)$ along the vertical z-axis by solving the PDE (1) online. The finite element model considers heat conduction, convection by the movement of the glass, radiation and the time dependent disturbances caused by the end of the cylinder.

\section{Concept for real-time Optimization of a spatially distributed temperature profile}

In our solution concept (Fig. 2) we propose a real-time nonlinear model predictive control of the temperature of a forming zone (here for glass forming processes). In most cases the temperature profile cannot be measured directly, hence it has to be estimated by a spatially distributed model. The one dimensional heat transfer PDE (1) has to be solved numerically for the temperature distribution $T(z, t)$, where $\mathrm{z}$ denotes the height and $\mathrm{t}$ the time. The time-dependent disturbances at the end of a cylinder are estimated by a disturbance model using the knowledge from previous productions to determine the model's parameters in a predictive way.

The NMPC approach is formulated as follows. The predicted values of the controlled variable $y(t)$ are collected in the vector $\hat{\boldsymbol{y}}$ for the timesteps from $t_{k} \ldots t_{k+n_{\mathrm{p}}}$, where $t_{k}$ is the actual time and $n_{\mathrm{p}}$ the prediction horizon. The predicted values are calculated by the model

$$
\hat{\boldsymbol{y}}=\boldsymbol{F}\left(\boldsymbol{x}_{k}, \Delta \boldsymbol{u}\right)
$$




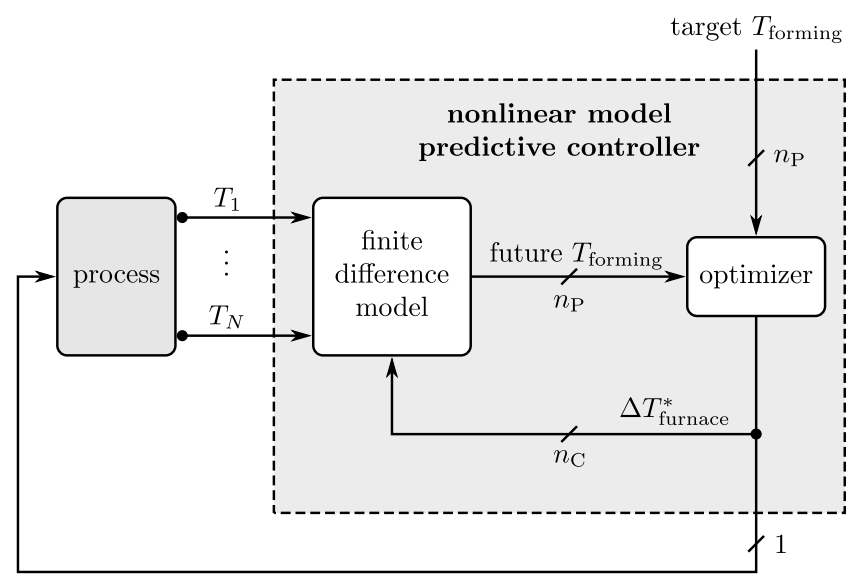

Fig. 2. NMPC temperature control scheme
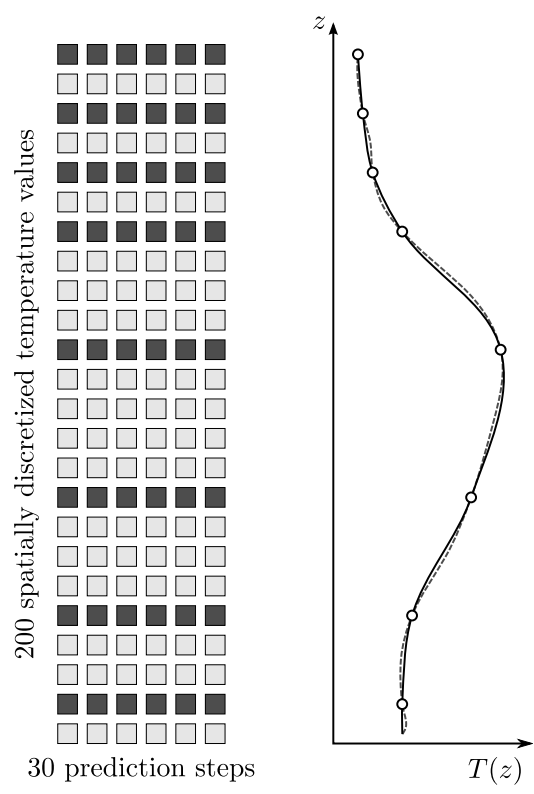

Fig. 3. Spline approximation 


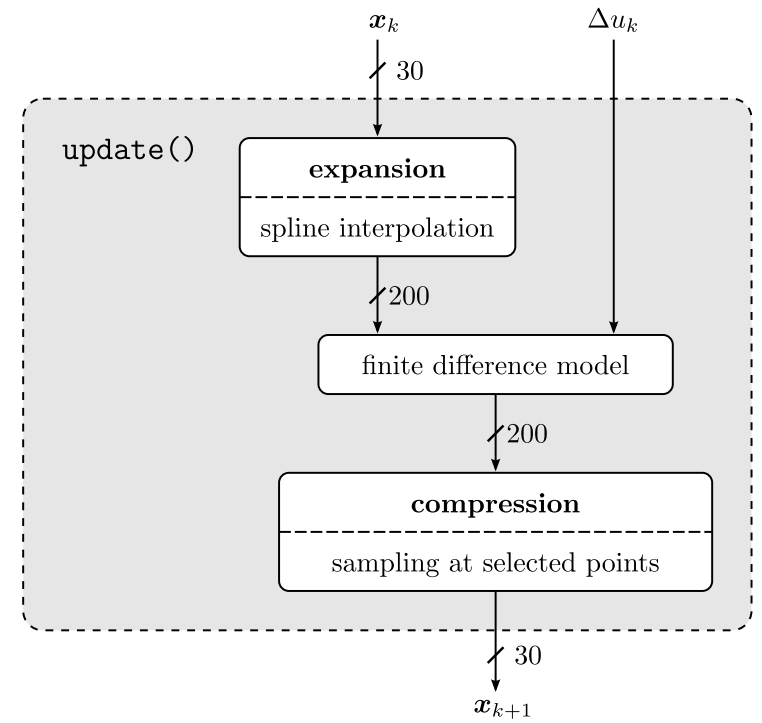

Fig. 4. HQP update step

where $\boldsymbol{x}_{k}$ denotes the actual state of the model and the vector $\Delta \boldsymbol{u}$ denotes all changes of the controlled variables from the actual time step $t_{k}$ to the prediction horizon (we set prediction horizon $n_{\mathrm{p}}$ equal control horizon $n_{\mathrm{p}}$ ). The optimization

$$
\begin{gathered}
J=(\boldsymbol{w}-\hat{\boldsymbol{y}})^{\top} \boldsymbol{Q}(\boldsymbol{w}-\hat{\boldsymbol{y}})+\Delta \boldsymbol{u}^{\top} \boldsymbol{R} \Delta \boldsymbol{u} \\
\Delta \boldsymbol{u}_{\mathrm{opt}}=\underset{\Delta \boldsymbol{u}}{\operatorname{argmin}} J
\end{gathered}
$$

is performed subject to

$$
\begin{aligned}
\Delta u_{\min } \leq \Delta u(k+j \mid k) \leq \Delta u_{\max } & j=0, \ldots, n_{\mathrm{C}}-1 \\
\hat{y}_{\min } \leq \hat{y}(k+j \mid k) \leq \hat{y}_{\max } & j=1, \ldots, n_{\mathrm{P}}
\end{aligned}
$$

In this formulation the changes of the manipulated variable $u$ are calculated. If necessary, also the absolute value $u$ could be considered directly in the optimization problem.

The proposed control concept has been implemented in a $\mathrm{C}++$ application by utilizing the software suite HQP (Huge Quadratic Programming), a solver for large scale nonlinear programming problems, to solve the arising nonlinear optimal control problems. In order to meet real-time requirements, in each sampling interval the full finite element discretization of the temperature profile is reduced considerably by a spline approximation (see Fig. 4 and 3). The spline 
approximation is admissible due to the temperature profile's smoothness. In our application the number of states could be reduced from 200 to 30 .

\section{Results}

We evaluated the control performance resulting with the proposed NMPC concept and compared it to a PI controller using previously recorded production data. It is shown that NMPC stabilizes the temperature of the forming zone much better than PI control. As can be seen in Fig. 5 with very small prediction and control horizon $\left(n_{\mathrm{p}}=n_{\mathrm{c}}=2\right)$ the performance of NMPC and PI control do not differ very much. In subplot 1 of Fig. 5 the deviation $e_{\text {forming }}$ from the reference value is shown for a production time of 40 minutes. In subplot 2 the change of the furnace temperature (manipulated variable) compared to the value at starting time $t=0 \mathrm{~min}$. can be seen (i.e., $\bar{T}_{\text {furnace }}(t)=T_{\text {furnace }}(t)-T_{\text {furnace }}(0)$ ). Subplot 3 shows the changes of furnace temperature in each sample time. These changes have been restricted to $\pm 5 \mathrm{~K}$ during the optimization in order to guarantee a smooth change of the furnace temperature. Finally, in subplot 4 the computation time for each sample interval $(30 \mathrm{~s})$ is shown. It can be seen that the computation time does not differ very much (about $2 \mathrm{~s}$ ). Fig. 6 shows the results with a much larger prediction and control horizon $\left(n_{\mathrm{p}}=n_{\mathrm{c}}=30\right)$. From subplot 1 it can be observerd that the control deviation with NMPC is much smaller compared to the results with PI control. Accordingly, the decrease of the furnace temperature (subplot 2) with NMPC starts much earlier. This is caused by the internal disturbance model which predicts the disturbances early enough. From subplot 3 it can be seen that the defined threshold for changes of the furnace temperature are reached for a period of about 10 minutes. In subplot 4 it can be seen that the computation is always smaller than the sample time of $30 \mathrm{~s}$, hence the real-time condition is satisfied. The computation time decreases strongly in the last 15 minutes of the production due to the shortened horizon.

The allowed parameter settings for a real-time application (e.g., control horizon, sampling period) have been determined. In Fig. 7 the computation time is plotted versus the prediction and control horizon $\left(n_{\mathrm{p}}, n_{\mathrm{c}}\right)$. The internal time discretization $d \tau$ of the finite element model $\left(n_{\mathrm{FDM}}=T_{\mathrm{s}} / d \tau\right)$ is used as parameter ( $T_{\mathrm{s}}$ : sample time). From Fig. 7 it is obvious that for a wide range of parameter values $n_{\mathrm{p}}, n_{\mathrm{c}}$ and $n_{\mathrm{FDM}}$ real-time application (computation time $<$ sample time $=30 \mathrm{~s})$ is possible.

Furthermore, simulation based application of the proposed NMPC scheme to a large number of historical runs showed that the concept is robust against model mismatch of the disturbance model. This property is very important for the industrial implementation. 

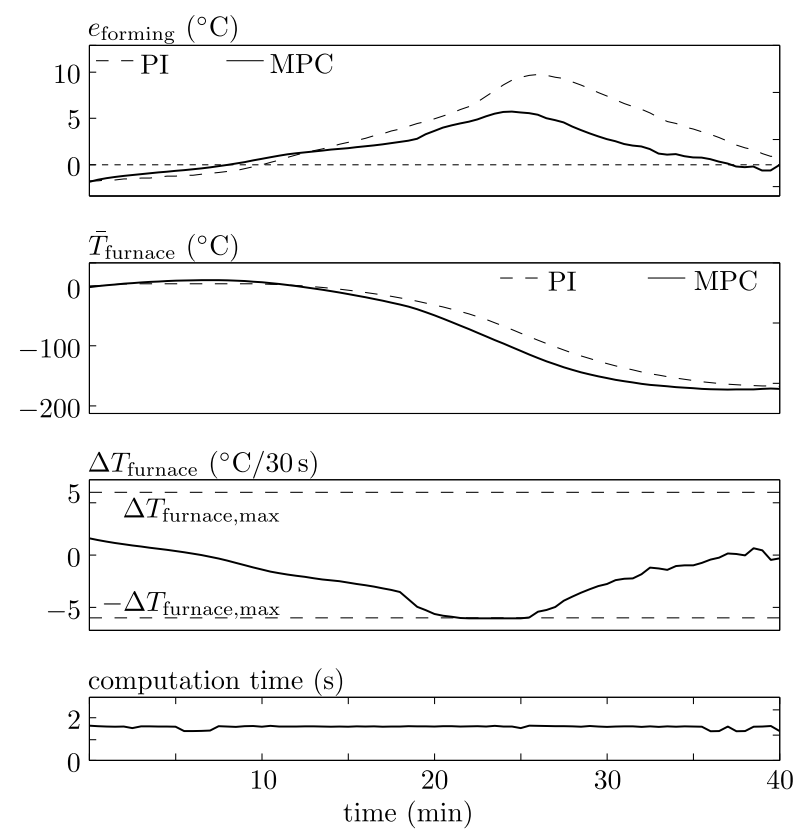

Fig. 5. Comparison NMPC and PI control $\left(n_{\mathrm{P}}=n_{\mathrm{C}}=2\right)$
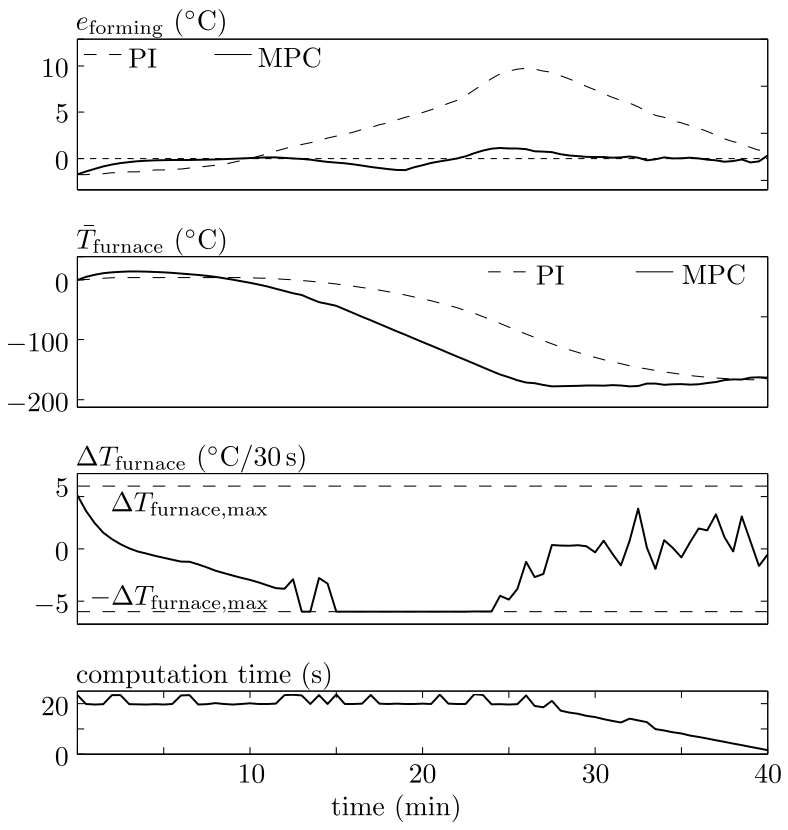

Fig. 6. Comparison NMPC and PI control $\left(n_{\mathrm{P}}=n_{\mathrm{C}}=30\right)$ 


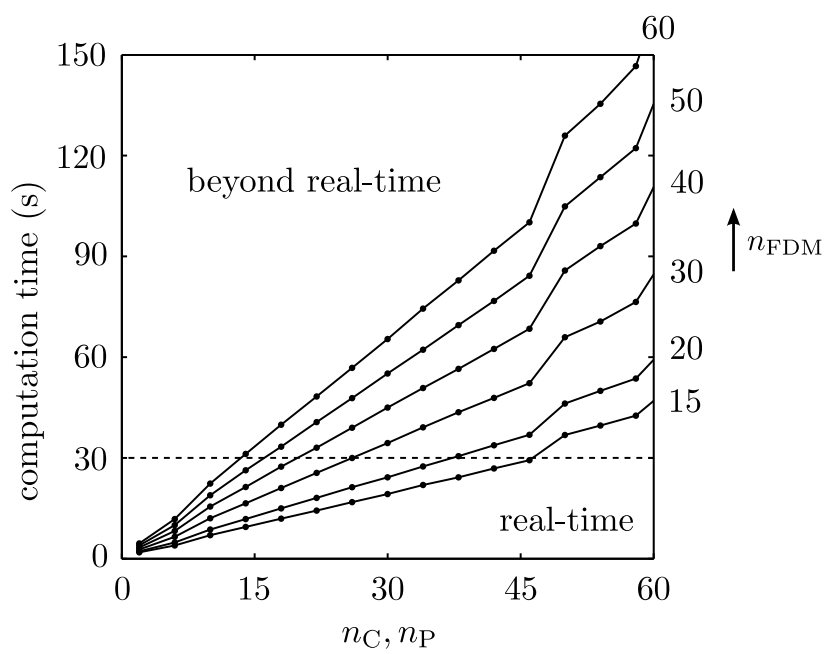

Fig. 7. Computation time vs. prediction horizon

\section{Conclusion}

In this paper a new approach for the real-time control of a spatially distributed temperature profile of an industrial glass forming process has been presented. A numerically efficient solution of the partial differential equation has been implemented. By means of the powerful solver HQP a real-time application could be achieved. It has been shown that with the proposed NMPC concept much better disturbance rejection and hence stabilization of the temperature profile than conventional PI control is achieved. The approach can easily be adapted to other forming processes where the temperature profile shall be controlled.

\section{References}

1. Glass Days 2005 - Analysis and Simulation of Processes in Glass Production and Processing, Kaiserslautern, Germany (April $14-15,2005)$

2. EU project PROMATCH (PROmoting and structuring Multidisciplinary Academic-industrial collaboration in research and Training trough SME teCHnology developers) (2008), http://promatch.ele.tue.nl/

3. Antoulas, A.C.: Approximation of Large-Scale Dynamical Systems. SIAM Press: Philadelphia (2005)

4. Benner, P., Sorensen, D.C., Mehrmann, V.: Dimension Reduction of Large-Scale Systems. Springer-Verlag Berlin (2005)

5. Bernard, T., Krol, O., Linke, H., Rauschenbach, T.: Optimal management of regional water supply systems using a reduced finite-element groundwater model. at - Automatisierungstechnik 57(12), 593-600 (2008) 
6. Bernard, T., Moghaddam, E.E.: Nonlinear model predictive control of a glass forming process based on a finite element model. In: IEEE Conference on Control Applications (CCA 2006). Munich, Germany (October 2006)

7. D'Amato, F.J.: Industrial application of a model predictive control solution for power plant startups. Proc. 2006 IEEE International Conference on Control Applications (2006)

8. Diehl, M., et al.: An efficient algorithm for nonlinear model predictive control of large-scale systems part 1. at - Automatisierungstechnik 50(12) (2002)

9. Diehl, M., et al.: An efficient algorithm for nonlinear model predictive control of large-scale systems part 2. at - Automatisierungstechnik 51(1) (2003)

10. Franke, R., Arnold, E.: The solver Omuses/HQP for structured largescale constrained optimization: algorithm, implementation and example application. Sixth SIAM Conference on Optimization (1999), http://hqp. sourceforge.net/index. html

11. Krause, D.: Mathematical Simulation in Glass Technology. Springer (2002)

12. Nagy, Z.K., Franke, R., Mahn, B., Allgöwer, F.: Real-time Implementation of Nonlinear Model Predictive Control of Batch Processes in an Industrial Framework, Freudenstadt, Germany. Proc. NMPC'05 (August 26 - 30, 2005) 